\title{
Effect of Switching from the Initial Direct Oral Anticoagulant to Another One on Exacerbation of Venous Thromboembolism in Patients with Cancer: A Retrospective Study
}

\author{
Takuya Oyakawa, MD, ${ }^{1}$ Nao Muraoka, MD, ${ }^{1}$ Kei lida, MD, ${ }^{1}$ and Masatoshi Kusuhara, MD, PhD ${ }^{2}$
}

Objective: To determine the effect of switching from the initial direct oral anticoagulant (DOAC) to another DOAC on exacerbation of deep vein thrombosis (DVT).

Materials and Methods: We retrospectively reviewed the data of patients with advanced cancer who experienced exacerbated DVT during initial treatment with DOAC due to new venous thromboembolism (VTE). After switching to another DOAC for VTE recurrence, changes in the thrombus and bleeding were evaluated for 3 months. Eighteen patients met these criteria. We compared the effect of anticoagulant switching on the switched-drug group in those 18 patients with the effect of no anticoagulant switching on the single-drug group of patients $(n=78)$ with a similar background.

Results: The recurrence rate of VTE in the switched-drug group was $6 \%$. Non-major bleeding occurred in $11 \%$ of patients. Recurrent VTE occurred in $6 \%$ of patients in both the switched-drug and single-drug groups, respectively [risk ratio (RR): $0.9,95 \%$ confidence interval $(\mathrm{Cl})$ : 0.11-7.6]. Non-major bleeding occurred in $11 \%$ and $14 \%$ of patients in the switched-drug and single-drug groups, respectively (RR: 0.79, 95\%Cl: 0.19-3.2).

Conclusion: Switching DOAC may be a treatment option for exacerbation of DVT in patients with advanced cancer.

${ }^{1}$ Division of Cardiology, Shizuoka Cancer Center, Shizuoka, Japan

${ }^{2}$ Research Institute, Shizuoka Cancer Center, Shizuoka, Japan

Received: June 21, 2018; Accepted: August 23, 2018

Corresponding author: Takuya Oyakawa, MD. Division of Cardiology, Shizuoka Cancer Center, 1007 Shimonagakubo, Nagaizumi-cho, Sunto-gun, Shizuoka 411-8777, Japan

Tel: +81-55-989-5222, Fax: +81-55-989-5783

E-mail: t.oyakawa@scchr.jp

(cc) BY-NC-SA (C2018 The Editorial Committee of Annals of Vascular Diseases. This article is distributed under the terms of the Creative Commons Attribution License, which permits use, distribution, and reproduction in any medium, provided the credit of the original work, a link to the license, and indication of any change are properly given, and the original work is not used for commercial purposes. Remixed or transformed contributions must be distributed under the same license as the original.
Keywords: recurrent venous thromboembolism, direct oral anticoagulant, anticoagulant switching, pulmonary thromboembolism, cardio-oncology

\section{Introduction}

The American College of Chest Physicians guideline states that in patients who have recurrent venous thromboembolism (VTE), while receiving warfarin therapy or direct oral anticoagulant (DOAC) therapy, treatment should be switched to a low-molecular-weight heparin (LMWH), at least temporarily. ${ }^{1)}$ Although LMWH is recommended, there is no insurance coverage for this treatment of VTE in Japan. Therefore, switching the anticoagulant to another DOAC or warfarin is considered for the recurrence of VTE when the patient is already receiving treatment with DOAC. No study in PubMed (National Center for Biotechnology Information) has been published about the effectiveness of switching the initial DOAC to another one (anticoagulant switching) for VTE.

Cancer is a risk factor for thrombosis, and patients with cancer are 4-7 times more likely to have a VTE than patients without cancer. ${ }^{2,3)}$ The recurrence rate of VTE is reported to be three times higher in patients with cancer than in patients without. ${ }^{4,5)}$ Furthermore, it is usually difficult to control the warfarin level of patients with cancer because of drug interactions and anorexia. ${ }^{6,7)}$

In this study, we evaluated the effect of anticoagulant switching on exacerbation of deep vein thrombosis (DVT) in patients with cancer receiving initial therapy with a DOAC.

\section{Materials and Methods}

We retrospectively reviewed the data of patients with advanced cancer who experienced exacerbated DVT during initial treatment with DOAC due to new VTE between September 2015 and October 2017 at Shizuoka Cancer Center. VTE was defined as proximal DVT and/or pulmo- 
nary thromboembolism (PE).

After anticoagulant switching, changes in the thrombus and bleeding were evaluated for 3 months. A patient in whom the thrombus increased or appeared new was regarded as having a recurrence of DVT. The thrombus was evaluated by echography or contrast-enhanced computed tomography (CT). We categorized bleeding as confirmed major bleeding and non-major bleeding. ${ }^{8,9)}$ DOAC was administered according to the drug package insert.

We compared the effect of anticoagulant switching on the switched-drug group in 18 patients with the effect of no anticoagulant switching in the single-drug group (firstline therapy with a DOAC) of another group of patients with a similar background that we analyzed previously using the same endpoints of the switched-drug group. ${ }^{10)}$ The endpoints were the bleeding rate and recurrence rate for 3 months after the start of treatment. Patients in whom thrombosis was not evaluated by echography or CT after 3 months were excluded from the assessment of VTE recurrence.

\section{Statistical analysis}

To analyze differences in patient background characteristics, Fisher's exact test or Wilcoxon's rank sum test was used. The difference between each patient group was evaluated using Fisher's exact test. Statistical significance was set at $\mathrm{p}<0.05$. The analysis was performed with JMP 9 (SAS Institute Inc., Cary, NC, USA).

\section{Results}

Among 18 patients, the median age was 67 years, mean ( \pm 2 standard deviation); body weight was 55.0 (12.5) $\mathrm{kg}$; and creatinine level was $0.70(0.26) \mathrm{mg} / \mathrm{dL}$. Fifteen patients had undergone chemotherapy, and eight were complicated with PE at the start of anticoagulant switching. Types of cancer were lung cancer $(n=6)$, pancreatic cancer $(n=3)$, colorectal cancer $(n=2)$, and other $(n=7)$. The recurrence of VTE with anticoagulant switching oc- curred in one patient $(6 \%)$. There was no incidence of major bleeding. Non-major bleeding occurred in two patients $(11 \%)$ (Table 1$)$. The treatment effects of anticoagulant switching were as follows: thrombus reduction, 10 patients; no thrombus change, seven patients; and thrombosis exacerbation, one patient (Table 2). Non-major bleeding included melena and hematuria. In patients with thrombus reduction or disappearance, DOAC was changed as follows: from apixaban to $60 \mathrm{mg}$ edoxaban $(n=1), 60 \mathrm{mg}$ edoxaban to apixaban $(\mathrm{n}=1), 30 \mathrm{mg}$ edoxaban to apixaban $(\mathrm{n}=5)$, rivaroxaban to $60 \mathrm{mg}$ edoxaban $(\mathrm{n}=1)$, and rivaroxaban to $30 \mathrm{mg}$ edoxaban $(\mathrm{n}=2)$. In patients without thrombus change, DOAC was changed as follows: from apixaban to $60 \mathrm{mg}$ edoxaban $(\mathrm{n}=2), 30 \mathrm{mg}$ edoxaban to apixaban $(\mathrm{n}=2)$, rivaroxaban to apixaban $(\mathrm{n}=1)$, and rivaroxaban to $30 \mathrm{mg}$ edoxaban $(\mathrm{n}=2)$. In patients with thrombus propagation, DOAC was changed from apixaban to $30 \mathrm{mg}$ edoxaban (Table 3 ).

Next, we compared the effect of anticoagulant switching on the switched-drug group with that of no anticoagulant switching on the single-drug group (first-line DOAC treatment) in our hospital. ${ }^{10)}$ Patient background charac-

Table 1 VTE recurrence and bleeding in second-line therapy after switching the direct oral anticoagulant $(\mathrm{N}=18)$

\begin{tabular}{lcr}
\hline & $\mathrm{n}$ & $\%$ \\
\hline VTE recurrence & 1 & 6 \\
Non-major bleeding & 2 & 11 \\
Major bleeding & 0 & 0 \\
\hline VTE. venous thromboembolism & &
\end{tabular}

VTE: venous thromboembolism

Table 2 Treatment effect after switching the initial DOAC to a second DOAC (anticoagulant switching)

\begin{tabular}{lrr}
\hline Treatment effect after switching the DOAC & $\mathrm{n}$ & $\%$ \\
\hline Thrombus reduction or disappearance & 10 & 56 \\
No thrombus change & 7 & 39 \\
Thrombus propagation & 1 & 6 \\
\hline
\end{tabular}

DOAC: direct oral anticoagulant

Table 3 Details of the initial and switched direct oral anticoagulant treatment

\begin{tabular}{|c|c|c|c|c|}
\hline & Initial anticoagulant & Switched anticoagulant & $\mathrm{n}$ & $\%$ \\
\hline \multirow[t]{5}{*}{ Thrombus reduction or disappearance } & Edoxaban $(60 \mathrm{mg})$ & Apixaban & 1 & 6 \\
\hline & Edoxaban (30 mg) & Apixaban & 5 & 28 \\
\hline & Rivaroxaban & Edoxaban (60 mg) & 1 & 6 \\
\hline & & Edoxaban (30 mg) & 2 & 11 \\
\hline & Apixaban & Edoxaban $(60 \mathrm{mg})$ & 1 & 6 \\
\hline \multirow[t]{4}{*}{ No thrombus change } & Edoxaban (30 mg) & Apixaban & 2 & 11 \\
\hline & Rivaroxaban & Edoxaban (30 mg) & 2 & 11 \\
\hline & & Apixaban & 1 & 6 \\
\hline & Apixaban & Edoxaban $(60 \mathrm{mg})$ & 2 & 11 \\
\hline Thrombus propagation & Apixaban & Edoxaban (30 mg) & 1 & 6 \\
\hline
\end{tabular}


Table 4 Comparison of patient background characteristics between the switched-drug and single-drug groups

\begin{tabular}{|c|c|c|c|c|}
\hline & & $\begin{array}{l}\text { Switched-drug group } \\
\qquad(n=18)\end{array}$ & $\begin{array}{l}\text { Single-drug group } \\
\qquad(n=78)\end{array}$ & p-value \\
\hline Age, years & Median (range) & $67(42-77)$ & $65.5(47-94)$ & 0.85 \\
\hline Sex & Male/female & $10 / 8$ & $40 / 38$ & 0.80 \\
\hline Body weight & Mean (standard deviation), kg & $55.0(12.5)$ & $57.7(10.3)$ & 0.30 \\
\hline Creatinine level & Mean (standard deviation), mg/dL & $0.70(0.26)$ & $0.70(0.20)$ & 0.93 \\
\hline Hemoglobin level & Mean (standard deviation), g/dL & $9.9(1.5)$ & $11.6(2.2)$ & 0.001 \\
\hline Serum platelet count & Mean (standard deviation), $10^{4} / \mu \mathrm{L}$ & $25.3(12.2)$ & $20.9(8.4)$ & 0.16 \\
\hline Chemotherapy & Treated/not treated & $15 / 3$ & $42 / 36$ & 0.03 \\
\hline Pulmonary thromboembolism & Yes/no & $8 / 10$ & $39 / 39$ & 0.80 \\
\hline
\end{tabular}

Table 5 Comparison of VTE recurrence and bleeding in the switched-drug and single-drug groups

A. VTE recurrence

\begin{tabular}{|c|c|c|c|c|}
\hline & $\begin{array}{l}\text { Switched-drug group } \\
\qquad(n=18)\end{array}$ & $\begin{array}{l}\text { Single-drug group } \\
\qquad(n=65)\end{array}$ & $\begin{array}{c}\text { Risk ratio } \\
(95 \% \text { confidence interval) }\end{array}$ & $\mathrm{p}$-value \\
\hline VTE recurrence & $6 \%$ & $6 \%$ & $0.90(0.11-7.6)$ & 1.00 \\
\hline \multicolumn{5}{|l|}{ B. Bleeding } \\
\hline & $\begin{array}{l}\text { Switched-drug group } \\
\qquad(n=18)\end{array}$ & $\begin{array}{l}\text { Single-drug group } \\
\qquad(n=78)\end{array}$ & $\begin{array}{c}\text { Risk ratio } \\
(95 \% \text { confidence interval) }\end{array}$ & $\mathrm{p}$-value \\
\hline Non-major bleeding & $11 \%$ & $14 \%$ & $0.79(0.19-3.2)$ & 1.00 \\
\hline Major bleeding & $0 \%$ & $0 \%$ & - & - \\
\hline
\end{tabular}

VTE: venous thromboembolism

teristics showed a significant difference in the hemoglobin level and history of chemotherapy (Table 4). Compared with the single-drug group, the switched-drug group had a lower hemoglobin value and more of them were undergoing chemotherapy. In the single-drug group, 65 patients were evaluated for the recurrence of VTE because the change in the thrombus was not evaluated at 3 months in 13 patients. Recurrent VTE in the switched-drug and single-drug groups occurred in $6 \%$ of patients [risk ratio (RR): 0.9, 95\% confidence interval (CI): 0.11-7.6] (Table $5 \mathrm{~A})$. Non-major bleeding occurred in $11 \%$ and $14 \%$ of patients in the switched-drug and single-drug groups, respectively (RR: 0.79, 95\%CI: 0.19-3.2). There were no significant statistical differences in data between these treatment groups. There was also no incidence of major bleeding (Table 5B).

\section{Discussion}

In our small retrospective cohort, the recurrence of VTE after anticoagulant switching was $6 \%$. The switched-drug group, which was treated using a different DOAC for VTE recurrence, showed similar results to those of the singledrug group for newly diagnosed VTE in the therapeutic effect and bleeding ratio. These findings suggested that switching DOAC may be effective for preventing the recurrence of VTE.

In atrial fibrillation, switching DOAC was effective for patients in whom left atrial thrombus developed during the oral administration of DOAC. ${ }^{11,12)}$ Pharmacological properties of each DOAC are different. ${ }^{13)}$ Therefore, patients with cancer, who usually have changes in renal function, body weight, and concomitant drugs, may achieve a therapeutically effective plasma level of anticoagulation by switching to a different DOAC.

Edoxaban is administered at a reduced dose for a body weight of $\leq 60 \mathrm{~kg}$. When the patient's body weight is $\leq 60 \mathrm{~kg}$, changing edoxaban to apixaban or rivaroxaban has the effect of increasing the DOAC dose. However, if the patient's body weight is $\leq 60 \mathrm{~kg}$, changing DOAC to edoxaban has the effect of reducing the DOAC dose. In this study, thrombus propagation was found when DOAC was switched to one with a reduced dose. The American College of Chest Physicians guideline states that in patients who have recurrent VTE and have been taking long-term LMWH, the LMWH dose should be increased by about one-quarter to one-third. ${ }^{1)}$ At the time of recurrence of VTE, increasing the doses of medication is recommended so it may be better to avoid switching DOAC to one with a reduced dose. In addition, patients who newly 
experienced non-major bleeding in the switched-drug group were all patients who were switched to a DOAC with an increased dose.

This study has some limitations. For VTE in patients with cancer, the recurrence of VTE was $7.9 \%$ with treatment with edoxaban. ${ }^{14)}$ As described earlier, the number of patients with VTE recurrence while receiving DOAC treatment was small, and the number of patients in this study was very small. In order to confirm the effect of anticoagulant switching, a comparison between switching from one DOAC to another and switching from DOAC to LMWH or warfarin is essential. In the future, prospective studies with more cases are needed.

\section{Conclusion}

In patients with advanced cancer and exacerbation of DVT, a thrombus recurrence rate of $6 \%$ was observed after anticoagulant switching for VTE. Switching DOAC may be a treatment option for exacerbation of DVT in patients with advanced cancer. With the advent of DOAC therapy, the options for VTE treatment have increased. It is necessary to confirm the effectiveness of secondary therapy for recurrent VTE, including switching DOACs.

\section{Disclosure Statement}

The authors declare no conflict of interest.

\section{Author Contributions}

Study conception: TO, KI

Analysis: TO

Investigation: TO, KI

Writing: TO

Critical review and revision: all authors

Final approval of the article: all authors

Accountability for all aspects of the work: all authors

\section{References}

1) Kearon C, Akl EA, Ornelas J, et al. Antithrombotic therapy for VTE disease: CHEST guideline and expert panel report. Chest 2016; 149: 315-52.
2) Heit JA, Silverstein MD, Mohr DN, et al. Risk factors for deep vein thrombosis and pulmonary embolism: a population-based case-control study. Arch Intern Med 2000; 160: 809-15.

3) Cronin-Fenton DP, Søndergaard F, Pedersen LA, et al. Hospitalisation for venous thromboembolism in cancer patients and the general population: a population-based cohort study in Denmark, 1997-2006. Br J Cancer 2010; 103: 947 53.

4) Hutten BA, Prins MH, Gent M, et al. Incidence of recurrent thromboembolic and bleeding complications among patients with venous thromboembolism in relation to both malignancy and achieved international normalized ratio: a retrospective analysis. J Clin Oncol 2000; 18: 3078-83.

5) Prandoni P, Lensing AW, Piccioli A, et al. Recurrent venous thromboembolism and bleeding complications during anticoagulant treatment in patients with cancer and venous thrombosis. Blood 2002; 100: 3484-8.

6) Gunes A, Coskun U, Boruban C, et al. Inhibitory effect of 5-fluorouracil on cytochrome P450 2C9 activity in cancer patients. Basic Clin Pharmacol Toxicol 2006; 98: 197-200.

7) Shah SR, Martin R, Dowell JE, et al. Comparison of the 5-fluorouracil-warfarin and capecitabine-warfarin drug interactions. Pharmacotherapy 2010; 30: 1259-65.

8) Schulman S, Kearon C; Subcommittee on Control of Anticoagulation of the Scientific and Standardization Committee of the International Society on Thrombosis and Haemostasis. Definition of major bleeding in clinical investigations of antihemostatic medicinal products in non-surgical patients. J Thromb Haemost 2005; 3: 692-4.

9) van Gogh investigators; Buller HR, Cohen AT, Davidson B, et al. Idraparinux versus standard therapy for venous thromboembolic disease. N Engl J Med 2007; 357: 1094-104.

10) Oyakawa T, Muraoka N, Iida K, et al. Direct oral anticoagulant for the treatment of venous thromboembolism in Japanese patients with cancer. Palliative Care Research 2017; 12: 175-82 (in Japanese).

11) Watanabe T, Shinoda Y, Ikeoka K, et al. Dabigatran therapy resulting in the resolution of rivaroxaban-resistant left atrial appendage thrombi in patients with atrial fibrillation. Intern Med 2017; 56: 1977-80.

12) Koyama T, Otsuka Y, Kawahara M, et al. A left atrial appendage thrombus that developed during prophylactic low-dose dabigatran treatment resolved after switching to apixaban. Clin Case Rep 2017; 5: 711-3.

13) Yeh CH, Fredenburgh JC, Weitz JI. Oral direct factor Xa inhibitors. Circ Res 2012; 111: 1069-78.

14) Raskob GE, van Es N, Verhamme P, et al. Edoxaban for the treatment of cancer-associated venous thromboembolism. N Engl J Med 2018; 378: 615-24. 